\title{
Intracranial Malignant Triton Tumor in Children: A Case Report
}

\author{
Xianwen $\mathrm{Hu}{ }^{(1)}{ }^{1}$, Xiaolan $\mathrm{He}^{2}$, Pan Wang ${ }^{1}$, Yujia Zou ${ }^{3}$, Dandan $\mathrm{Li}^{4}$ and Jiong Cai ${ }^{1,{ }^{*}}$ \\ ${ }^{1}$ Department of Nuclear Medicine, Affiliated Hospital of Zunyi Medical University, Zunyi, China \\ ${ }^{2}$ Department of Pathology, Guizhou Provincial People's Hospital, Guiyang, China \\ ${ }^{3}$ Translational Medical Center, Affiliated Hospital of Zunyi Medical University, Zunyi, China \\ ${ }^{4}$ Department of Obstetrics and Gynecology, Zunyi Hospital of Traditional Chinese Medicine, Zunyi, China \\ "Corresponding author: Department of Nuclear Medicine, Affiliated Hospital of Zunyi Medical University, Zunyi, China. Email: jiongcai@sina.com
}

Received 2020 June 06; Revised 2020 December 28; Accepted 2021 January 03.

\begin{abstract}
Malignant triton tumor (MTT) is a rare, aggressive type of tumor that commonly originates from mesenchymal tissue and is mainly found in adults. Herein, we report a single case of a 12-year-old boy diagnosed with intracranial MTT. This report presents the clinical features and imaging findings of MTT. The 12-year-old patient consulted the hospital due to intermittent dizziness and headache. Computed tomography (CT) showed low-density space-occupying lesion in the left parietal fossa. Magnetic resonance imaging(MRI) showed a mass shadow with slightly long T1 and long T2 signal intensity in the same area. Contrast enhanced MRI (CE-MRI) showed obvious enhancement of the lesion. He was diagnosed with meningioma and underwent surgery. Postoperative histopathological examination diagnosed the lesion as MTT. Two months after the operation, CT examination showed tumor recurrence. He then underwent local radiotherapy and chemotherapy, and unfortunately, died six months later. Intracranial MTT should be considered as one of the differential diagnoses of intracranial meningiomas. CT and MRI are of great significance in the identification of lesion location, invasion range, and degree of malignancy. Final diagnosis of intracranial MTT requires histopathological examination. MTT has a poor prognosis, and surgical resection of the tumor is the preferred treatment. Intervention after early diagnosis is the key to improve the outcome of patients.
\end{abstract}

Keywords: Malignant Triton Tumor, Intracranial, Children, Case Report

\section{Introduction}

Malignant triton tumor (MTT) is an aggressive type of tumor that originates from mesenchymal tissue and has a low incidence. It is classified as a subtype of malignant nerve sheath tumors containing elements of rhabdomyosarcoma. As a poorly differentiated tumor, MTT is prone to local recurrence and distant metastasis. It commonly arises from a peripheral nerve in the neck, extremities or trunk, while it is rarely found in the cranial temporal and parietal fossa, viscera, mediastinum and retroperitoneum (1). Recently, Dias et al. (cited in Zhao et al.) (2) have reported an uncommon case of cerebral MTT in Brazil. In addition, most MTT cases are reported in adults. Herein, we report a 12-year-old boy who went to a local hospital with a headache, and he underwent a series of imaging examinations and was diagnosed with meningioma. Subsequently, the patient underwent surgery, and postoperative pathological biopsy diagnosed the lesion as MTT. The purpose of this report is to introduce the clinical and imaging manifestations of MTT and to comprehensively analyze the diagnosis, differential diagnosis of MTT in combination with relevant literature.

\section{Case Presentation}

A 12-year-old boy was admitted to the outpatient department of the local hospital for intermittent dizziness and headache for 1 week. His family members denied the history of tuberculosis, tumor, infectious disease, and genetic disease. The patient has no uncomfortable physical symptoms other than lethargy. There was no bleeding on the skin of the whole body, and no palpable lymph node enlargement in the neck, armpit and groin. Physical examination of the patient's chest and abdomen showed no obvious abnormalities. Blood routine, stool routine, urine routine and tumor markers values were all within the normal range. The patient underwent computed tomography (CT) and magnetic resonance imaging (MRI) tests subsequently, CT images showed a low-density space-occupying lesion in the left parietal fossa (Figure 1A), and MRI showed the lesion with abnormal T1 and T2 signal intensity in the same area (Figure $1 \mathrm{~B}$ and $\mathrm{C}$ ), which was significantly enhanced on contrast-enhanced magnetic resonance imag- 
ing (CE-MRI) (Figure 1D and E). Based on the above imaging findings, the radiologists diagnosed the patient with meningioma; thereafter, the patient was transferred to the neurosurgery department of our hospital for surgery. Postoperative tumor tissue was sent to the pathology department for pathological section analysis. Hematoxylin \& eosin (H\&E) staining revealed extensive high mitosis (Figure 2A). Immunohistochemical staining for SOX10, NSE, GFAP were negative, while immunohistochemical staining with antibody indicated focal positive expression for S-100 (Figure 2B), and partial cells expression for myogenin (Figure $2 \mathrm{C}$ ). In addition, the myelin essential protein (MBP), Vimentin, Pgp9.5, CD57, CD56, CD99, Caldesmon, BCL-2, P53, SJLA were all positive. Part of tumor cells expressed Desmin, MyoD1, Myogenin, Calponin, and CD34. The ki-67 index was elevated (about $80 \%$ positive), and the STAT- 6 status was equivocal. Based on these data, the final pathological diagnosis suggested a malignant triton tumor (malignant peripheral nerve sheath tumor with rhabdomyoblastic differentiation). CT examination two months after the operation showed recurrence of the lesion (Figure 3), and he then underwent local radiotherapy and 6courses of chemotherapy in four months with ifosfamide, carboplatin and fluorouracil. However, platelet, erythrocyte and granulocyte decreased to different degrees during chemotherapy, and he died six months later.

\section{Discussion}

MTT was first reported in the 1930s and Woodruff first used the name "malignant triton tumor", inspired by Locatelli's experiments (3). MTT is an extremely rare malignant peripheral nerve sheath tumor with rapid growth and poor prognosis $(4,5)$. Histologically, MTT originates from malignant Schwann cells of peripheral nerves with heterogeneous differentiated striated muscle cells, i.e., glial cells in the peripheral nervous system (6). It is an advanced form of sarcoma, which seems to have a higher incidence in males than females and is relatively uncommon in children. More than half of MTT cases are associated with neurofibromatosis type 1 (NF-1) $(7,8)$. Furthermore, genetic analysis revealed some autosomal aberrations could lead to the occurrence and recurrence of MTT (9).

Imaging examinations have important clinical significance for the diagnosis of intracranial MTT. Common imaging tests include CT and MRI. CT is more valuable than MRI in showing calcification of tumor tissue and determining whether the tumor invades adjacent bone. MRI could clearly show not only the lesion location, size, shape and infiltration range, but it also gives important hints on the pathological components contained in the tumor.
On MRI images, most MTTs showed low signal intensity on T1 weighted imaging (T1WI) and high signal intensity on T2WI, and the lesions were significantly enhanced on T1WI of CE-MRI. Due to the high degree of malignancy and rapid progression of the disease, cystic necrosis in the tumor often occurs, resulting in uneven mass density/signal intensity (10). The radiographic findings of our patient were consistent with the above literature (10). On the T1WI of MRI, the lump showed slightly low signal intensity and blurred boundary with surrounding normal brain tissue, the solid portion of the mass and surrounding edema area presented high signal intensity on the T2WI of MRI, and nodular necrotic foci with long T1 signal intensity could be seen in the mass. Moreover, curved line-like low signal intensity shadows are visible on T2WI. Nevertheless, due to its rarity, no specific imaging findings have been identified for the diagnosis of the disease. The disease was misdiagnosed as meningioma by radiologists before surgery. We analyzed the reasons for misdiagnosis as the following: first of all, the mass was connected to the falx cerebri in a broad base on MRI, presenting a typical "D"-like change, and the boundary with the surrounding normal brain tissue was relatively clear, so an intracranial.

extraparenchymal tumor was considered, while meningioma is the most common intracranial tumor. Second, the MRI manifestations of meningiomas were similar to those of MTT, and most of them also showed short or equal T1 signal intensity and equal or long T2 signal intensity (7). Another more important reason for misdiagnosis was our radiologists' high confidence in the diagnosis of this lesion, after routine T1WI, T2WI and CE-MRI, MRI diffusion-weighted imaging (DWI) and magnetic resonance spectroscopy (MRS) analysis were not performed, leading to the absence of more imaging evidence.

The final diagnosis of intracranial MTT depends on histopathological examination. Microscopically, the tumor tissues were arranged in fasciculus and reticulosa, the fasciculus was malignant peripheral nerve cells, and a few pleomorphic cells scattered in the reticulosa were components of rhabdomytic tumors (11). The positive immunohistochemical results of S-100, Leu7, NSE, and myelin basic protein (MBP) confirmed the existence of nerve sheath cell components, and the positive results of desmin, myoglobin, and myosin suggested the presence of rhabdosarcoma components, the positive of S-100, desmin, and myoglobin, in particular, can be clearly diagnosed, and S-100 is generally weakly positive and distributed in MTT (11). In addition to the positive expression of these specific tumor proteins, Pgp9.5, CD57, CD56, CD99, Caldesmon, Bcl-2, P53, and SJLA were also positive in the patient we reported, confirming the malignant nature of the tumor. 

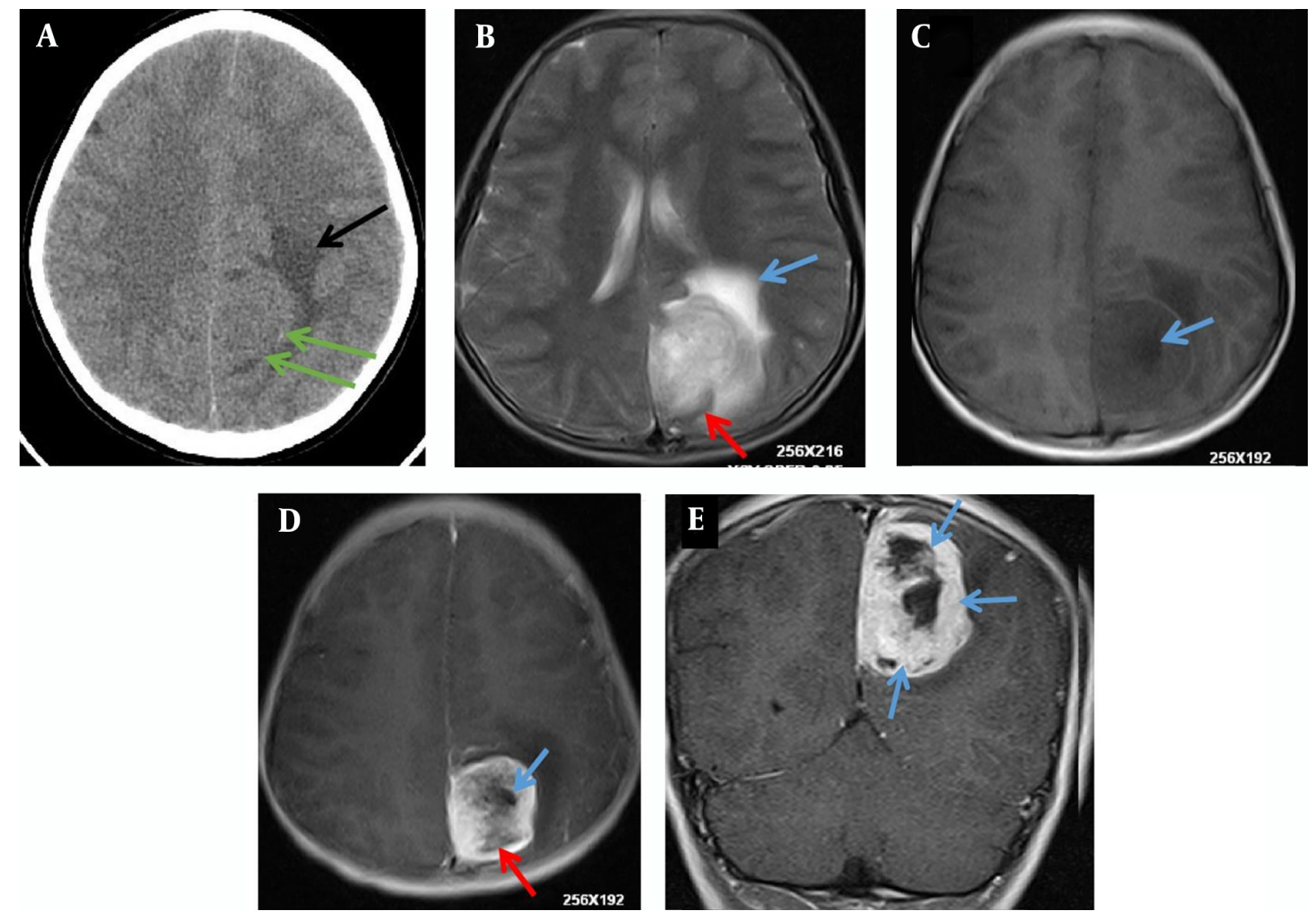

Figure 1. A 12-year-old boy with intermittent dizziness and headache for one week. Preoperative CT and MRI images of the patient. A, Axial CT scan shows an equal and slightly low-density mass shadow (green arrows) near the falx cerebri at the top of the left side, with unclear boundaries and low-density edema (black arrow); B, T2 weighted imaging (T2WI) of axial MRI shows uneven high signal intensity mass on the left side of the cerebral falx, line-like low signal intensity shadows were seen in the posterior margin of the mass (red arrow), and patchy higher signal intensity edema area in the front of the mass (blue arrow), C, T1 weighted imaging (T1WI) of axial MRI shows low and slightly low signal intensity in the lesion, with nodular necrosis with lower signal intensity (blue arrow); D, Axial TiWI of contrast-enhanced (CE)-MRI shows obvious enhancement of lesions, but no enhancement of nodular (blue arrow) and line like (red arrow) low signal intensity necrosis foci; E, Coronal T1WI of CE-MRI reveals multiple nodular, low signal intensity, and non-enhanced necrotic areas of unequal size (blue arrows).

According to the clinicopathological features and imaging findings, intracranial MTT needs to be differentiated from meningiomas, hemangiopericytoma, and other intracranial neurogenic tumors. Meningioma is a common tumor of central nervous system in adults, with a lower incidence in children and a higher incidence in women than in men. On CT images, meningiomas are usually spherical or hemispherical mass shadows with equal or high density (12), and the boundary between meningiomas and surrounding brain tissues is relatively clear. On MRI images, benign meningiomas are less prone to low signal intensity cystic degeneration and necrosis (13), while mesovariant meningiomas often show uneven signal intensity, can grow across the midline, and can destroy adjacent skulls; therefore, it is difficult to distinguish it from MTT. One study (10) suggested that the presence of annular or line-like low signal intensity in tumors with high signal intensity on the T2WI may be characteristic of MTT, which is associated with accelerated tumor progression and liquefied-necrosis. Intracranial hemangiopericytoma, as a rare mesenchymal tumor with imaging findings similar to meningiomas, should also be on the list of differential diagnoses for MTT. Hemangiopericytoma is a highly invasive malignant tumor with relatively severe edema, space occupying signs and cerebral hernia on CT and MRI images (14), which usually has uneven density/signal intensity due to hemorrhage, and cystic necrosis. Hemangiopericytomas are rich in tumor vessels and show tortuous vascular flow void signal intensity shadows on MRI, which are different from smooth curves or linear separation of low signal intensity shadows in MTT. In addition, angiopericytoma usually occurs at an older age compared to MTT (15). As a subtype of malignant schwannoma, the differentiation of MTT from other neurogenic tumors such as benign 

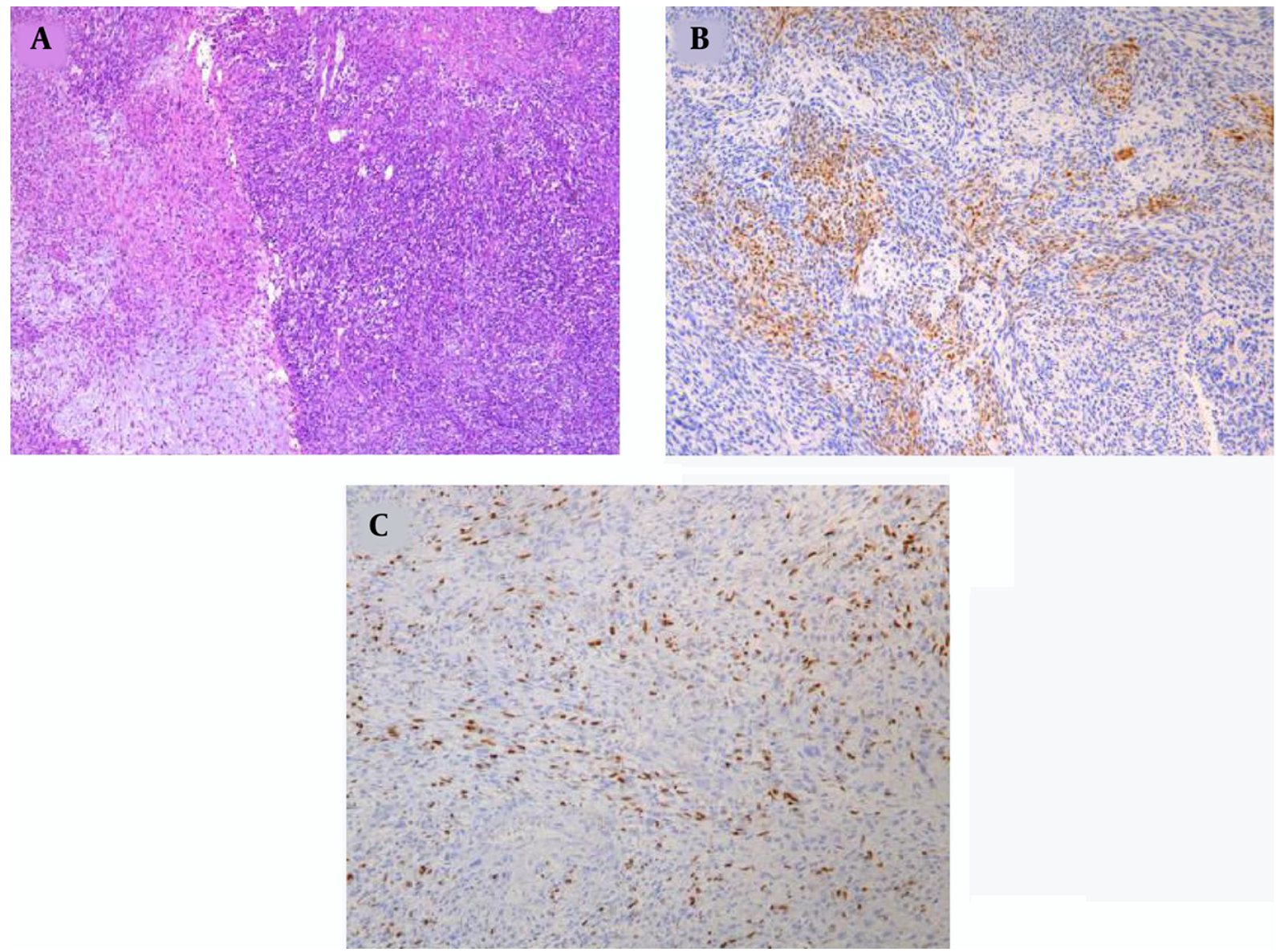

Figure 2. Pathology and immunohistochemical examination of the tissue. A, Hematoxyline and Eosin staining showed extensive high mitosis, $\times 100$; B, Immunohistochemical staining with antibody demonstrates focal positive expression for S-100; C, Partial cell expression for myogenin.

schwannoma and malignant schwannoma should also be included. The occurrence of intracranial schwannoma is closely related to the distribution of intracranial nerves, most of which are located in the cerebellopontine angle. Primary intracranial temporal and apical fossa schwannomas are rare, and some scholars (16) called them substantia neurilemmoma of the brain, of which pathogenesis are similar to MTT, may be related to schwann cells derived from perivascular plexus, pluripotent stromal cells or neural crest cells derived from ectopia (17). The imaging findings of neurilemmoma in the temporal and apical fossa are also similar to those of MTT. Benign schwannoma has a clearer boundary than malignant schwannoma, and both of them could present as nodular density/signal intensity abnormal cystic necrosis foci in the spindle or spherical mass, so their differential diagnosis with MTT could only be made by immunohistochemistry, and positivity of desmin and myoglobin is the key to identification. In addition, intracranial MTT has clear boundaries with normal brain tissue, so it is not difficult to differentiate from glioma and other solid brain tumors.

Because of the rarity of intracranial MTT, the World Health Organization has not yet established uniform treatment standards and guidelines. Clinically, the treatment of intracranial MTT usually follows the treatment regimen of other intracranial malignant tumors, and the main treatment for intracranial MTT reported worldwide is total tumor resection. Partial resection could be tried for tumors that cannot be completely removed due to large tumor size or invasion of important blood vessels. Radiotherapy and chemotherapy were used after surgery, common chemotherapy drugs include ifosfamide, actinomycin, carboplatin and fluorouracil, isotretinoin and interferon- $\alpha$, but its curative effect has not been clinically proven, and it is easy to relapse and metastasize in a short time, so the prognosis of intracranial MTT is very poor (7). Our patient 

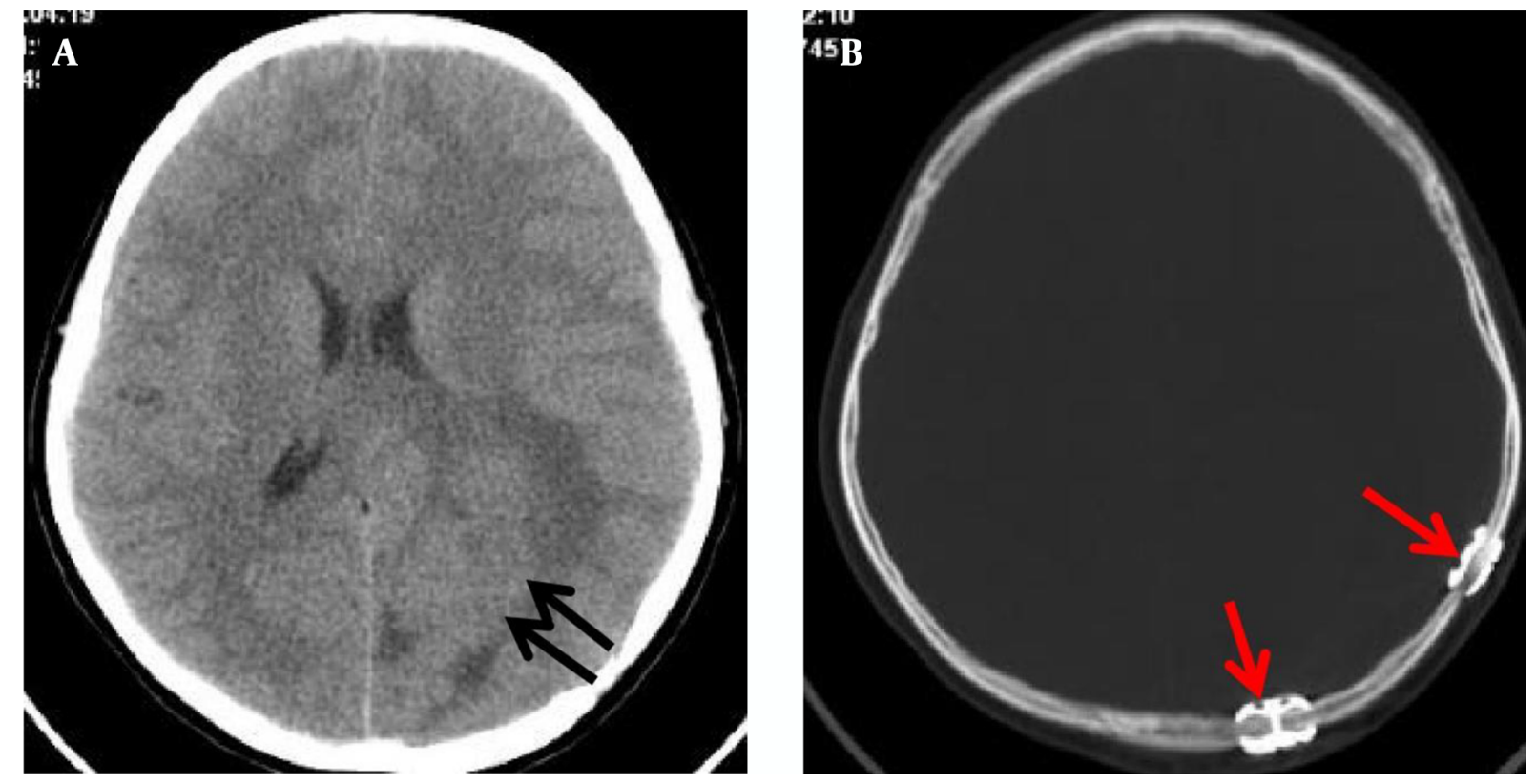

Figure 3. CT examination two months after surgery. A, Tissue window of brain CT shows recurrence of the lesion (black arrows); B, Bone window shows postoperative changes in the left parietal bone (red arrows).

had relapsed just two months after surgery to remove the mass, and had also received radiation and chemotherapy. Unfortunately, the patient died because of complications after chemoradiotherapy six months later.

In summary, imaging examination plays an important role in intracranial MTT diagnosis, especially in MRI examination, arc-shaped or line-shaped low signal intensity shadows found in tumor tissues with high signal intensity in T2WI have important diagnostic significance for MTT diagnosis. Intracranial MTT is relatively rare, but it still needs to be considered as one of the differential diagnoses of a common intracranial tumor - meningioma. The final diagnosis of MTT requires pathological and immunohistochemical examination. Positive staining of S-100, desmin, and myoglobin confirmed the diagnosis. However, intracranial MTT has a poor prognosis. Surgical resection of the tumor after early diagnosis, combined with radiotherapy and chemotherapy, is the key to prolong the lifespan of patients.

\section{Footnotes}

Authors' Contributions: HXW and WP conceived the case statement and drafted the manuscript. HXW and LD collected the patient's data and performed the follow-up. CJ and HXW wrote the manuscript. HXL wrote the commentaries below pathological pictures. CJ and ZY revised the manuscript. The authors read and approved the final manuscript.

Conflict of Interests: The authors state that there are no conflicts of interest.

Funding/Support: This study was funded by the National Natural Science Foundation of the Peoples Republic of China, NSFC (grant numbers: 81571712), Zunyi Medical College Research Start Fund 2018ZYFY03.

Informed Consent: Written informed consent was obtained from the patients family for publication of this case report.

\section{References}

1. Dias AB, Cambruzzi E, Giugno CS, Miranda de Curtis R, Faria do Amaral LL, Lenhardt R. Teaching NeuroImages: Intracranial malignant triton tumor: An uncommon location of a rare tumor. Neurology. 2019;92(12):e1401-2. doi: 10.1212/WNL.0000000000007144. [PubMed: 30886081].

2. Zhao A, Ding D, Li X, Wang J. Malignant Triton Tumor in a Child: Case Report and Literature Review. Cancer Manag Res. 2019;11:1075966. doi: 10.2147/CMAR.S221110. [PubMed: 31920385]. [PubMed Central: PMC6935315].

3. Woodruff JM, Chernik NL, Smith MC, Millett WB, Foote FJ. Peripheral nerve tumors with rhabdomyosarcomatous differentiation (malignant "Triton" tumors). Cancer. 1973;32(2):426-39. doi: 10.1002/10970142(197308)32:2<426::aid-cncr2820320221>3.0.co;2-w. [PubMed: 4198700].

4. Prieto R, Pascual JM, Garcia-Cabezas MA, Lopez-Barea F, Barrios L, Gonzalez-Llanos F. Low-grade malignant triton tumor in the lum- 
bar spine: a rare variant of malignant peripheral nerve sheath tumor with rhabdomyoblastic differentiation. Neuropathology. 2012;32(2):180-9. doi: 10.1111/j.1440-1789.2011.01238.x. [PubMed: 21732991].

5. Bian Y, Yongbo X, Xi Z, Zhao D, Wu H, Liu Y. A series of 10 malignant triton tumors in one institution. Medicine (Baltimore). 2019;98(36). e16797. doi: 10.1097/MD.0000000000016797. [PubMed: 31490366]. [PubMed Central: PMC6739022].

6. Qiao J, Ya L, Sun F, Yu X, Yu T, Tang K. Malignant triton tumor of the prostate: a case report. Int J Clin Exp Med. 2017;10(4):7308-11.

7. Li G, Liu C, Liu Y, Xu F, Su Z, Wang Y, et al. Analysis of clinical features and prognosis of malignant triton tumor: A report of two cases and literature review. Oncol Lett. 2015;10(6):3551-6. doi: 10.3892/ol.2015.3762. [PubMed: 26788168]. [PubMed Central: PMC4665219].

8. McConnell YJ, Giacomantonio CA. Malignant triton tumors-complete surgical resection and adjuvant radiotherapy associated with improved survival. J Surg Oncol. 2012;106(1):51-6. doi: 10.1002/jso.23042. [PubMed: 22253011].

9. Koutsimpelas D, Brieger J, Heinrich U, Torzewski M, Sommer C, Mann WJ. Cytogenetic analysis of a malignant triton tumour by comparative genomic hybridization (CGH) and review of the literature. Eur Arch Otorhinolaryngol. 2011;268(9):1391-6. doi: 10.1007/s00405-0111658-z. [PubMed: 21643932].

10. Lin L, Chen X, Jiang R, Zhong T, Du X, Xu G, et al. Differenti- ation between vestibular schwannomas and meningiomas with atypical appearance using diffusion kurtosis imaging and threedimensional arterial spin labeling imaging. EurJRadiol. 2018;109:13-8. doi: 10.1016/j.ejrad.2018.10.009. [PubMed: 30527294].

11. Deguchi S, Nakashima K, Nakasu Y, Mitsuya K, Hayashi N, Ito I, et al. A practical predictor of the growth potential of benign meningiomas: Hypointensity of surface layer in T2-weighted magnetic resonance imaging. Clin Imaging. 2020;62:10-6. doi: 10.1016/j.clinimag.2020.01.009. [PubMed: 32018148].

12. Chai W. CT diagnosis of meningioma in 40 cases. Shanghai Med Imaging. 2010;19(1):66-8.

13. Shijo M, Honda H, Koyama S, Ishitsuka K, Maeda K, Kuroda J, et al. Dura mater graft-associated Creutzfeldt-Jakob disease with 30-year incubation period. Neuropathology. 2017;37(3):275-81. doi: 10.1111/neup.12359. [PubMed: 27925304].

14. Chen W, Liu J. Comparative study on CT and MRI imaging characteristics of hemangiopericytoma of central nervous system. Shanxi J Med. 2020;49(10):1224-5.

15. Chen M, Li F, Liu CY, et al. Study on imaging features of intracranial hemanyiopericytoma correlated with pathology. Radiol Pract. 2014;29(1):49-52.

16. Song R, Wang Z. Imaging findings of schwannoma in intracranial non-pontocerebellar horn area. J Guangxi Med Univ. 2015;32(5):805-7.

17. Ouyang X, Mei K. Clinicopathologic analysis of schwannoma in brain parenchyma. Chin J Clin. 2015;2(9):405-8. 